\title{
Ion molecule reactions in vinyl fluoride by photoionization. Effects of vibrational excitation on major reaction pathways*
}

\author{
Ashley D.Williamson and J. L. Beauchamp ${ }^{\dagger}$ \\ Arthur Amos Noyes Laboratory of Chemical Physics, California Institute of Technology, Pasadena, \\ California 91125 \\ (Received 15 October 1975) \\ Photoionization methods have been used to study the reactions of the molecular ion in vinyl fluoride to \\ yield the ionic products $\mathrm{C}_{3} \mathrm{H}_{3} \mathrm{~F}_{2}^{+}, \mathrm{C}_{3} \mathrm{H}_{4} \mathrm{~F}^{+}$, and $\mathrm{C}_{3} \mathrm{H}_{5}^{+}$. Quantitative measurements are reported of the effect \\ of the vibrational state of the reactant ion on the product distribution and overall reaction cross section. \\ Reaction cross sections for all three channels decrease with reactant internal energy. The effect on the \\ reaction pathway producing $\mathrm{C}_{3} \mathrm{H}_{3} \mathrm{~F}_{2}^{+}$is especially pronounced, with $0.18 \mathrm{eV}$ of vibrational excitation being \\ sufficient to reduce the reaction probability by $75 \%$. Deactivation of vibrationally excited reactant ions \\ competes with the reaction and is shown to be an efficient process.
}

\section{INTRODUCTION}

At pressures low enough to avoid collisional stabilization of the excited intermediate, the molecular ion in vinyl fluoride undergoes Reactions (1)-(3). The enthalpy of Reaction (1) is calculated

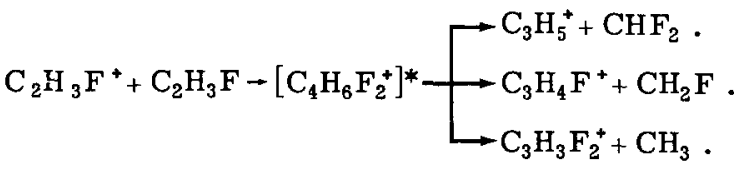

to be $-9 \mathrm{kcal} / \mathrm{mole}$; the thermochemistry of Reactions (2) and (3) is not as well characterized, but they have been estimated to be exothermic by 12 and $16 \mathrm{kcal} /$ mole, respectively. ${ }^{1}$

In recent years several groups have studied Reactions (1)-(3) using electron impact ionization, with differing conclusions concerning the kinetics and mechanism of these reactions. ${ }^{2-5}$ These studies indicate that the overall reaction rate and product distribution are affected by changing the ionizing electron energy and thus the internal energy distribution of the reactant ion. ${ }^{2,4}$ Beyond this general agreement, different mechanistic conclusions seem to be supported by different experimental techniques. Using a medium-pressure single source mass spectrometer, Herman and Harrison $^{2}$ and McAskill and Harrison ${ }^{3}$ observe clear indications of mixed second-order and third-order kinetics, each mechanism showing a characteristic product distribution. The ion cyclotron resonance (ICR) studies of O'Malley and Jennings ${ }^{4}$ and Anicich and Bowers ${ }^{5}$ show only second order kinetics, with a product distribution nearly identical to the third-order distribution of the single source studies. More recently Sieck et al. ${ }^{6}$ have studied Reactions (1)-(3) using photoionization by rare gas resonance lamps. Their data indicate that ions formed within $0.27 \mathrm{eV}$ of threshold react with a product distribution similar to the ICR and single source third-order values, while ions formed $1.3 \mathrm{eV}$ above threshold show initial product distributions and decay kinetics similar to those of the medium pressure instruments. Their results show that vibrational excitation alone is sufficient to cause the effects on the reaction kinetics.
The mixed kinetics of the single source data have been interpreted in terms of bimolecular steps using the assumption that the overall rate and product distribution for Reactions (1)-(3) are strongly affected by excess energy in the reactant ions. ${ }^{2,3,5}$ The competition between reaction and deactivation of ions in different energy states then leads to mixed kinetics and a product ratio that varies with the extent of reaction. The data of McAskill and Harrison ${ }^{3}$ could be fit with a model that assumed the distribution of initial states could be replaced by one composite excited state with a single effective rate of reaction and an effective deactivation rate. However, the ICR experiments could not be fit by this model without the assumption that all reactant ions were formed without excess excitation.

In view of the past interest in the vinyl fluoride sys tem, we have undertaken a study to further clarify the dynamics of these reactions. Using the techniques of photoionization mass spectrometry, we have made quantitative measurements of the dependence of Reactions (1)-(3) on the vibrational state of the reactant ion. The present work is an extension of our earlier study of ion-molecule reactions in ethylene ${ }^{7}$ and uses the techniques of that study. The use of photoionization mass spectrometry for investigating ion-molecule reactions has been recently reviewed by Chupka. ${ }^{8}$

\section{EXPERIMENTAL}

The photoionization mass spectrometer used in this study has been described elsewhere. ${ }^{7}$ The instrument has been modified slightly to facilitate data collection. The mass selected ions are presently detected by a Bendix model 4501 channeltron electron multiplier operated in a pulse-counting mode. The light detecting system was replaced by a scintillator-photomultiplier combination. A glass plate coated with sodium salicylate was placed in the back of the sample chamber. Fluorescence from the salicylate phosphor is detected by a small photomultiplier (RCA model 8571) mounted inside the vacuum chamber. The photomultiplier current is measured by a Cary 401 vibrating reed electrometer, whose output is filtered and fed to a voltage to frequency converter. Digitized ion and photon signals 
are then counted by a Hewlett-Packard 5243 counter operated in ratio mode; this method yields a direct output proportional to the photoionization efficiency of the ion under study and assures that counting statistics remain independent of the ionizing light intensity. In order to correct for variations in the quantum efficiency of the sodium salycilate phosphor, photoionization efficiency measurements on nitric oxide were made over the energy range of the present study. These data were compared with the absolute photoionization cross section data of Watenabe et al. ${ }^{9}$ to obtain a measurement of the relative quantum efficiency of our phosphor over the wavelength range $1200-1100 \AA$ and in turn to correct the photoionization efficiency data for vinyl fluoride and its ionic products.

Other modifications include addition of a servo system to control the wavelength drive of the monochromator and a small programmable controller for data collection. The monochromator servo system uses a stepping motor and an optical encoder mounted on the grating drive train of the monochromator to sense and adjust the position of the grating to a wavelength accuracy of $0.1 \AA$. The programmable controller supervises the data collection and allows up to ten masses to be sequentially monitored by the mass spectrometer.

Corrections for the mass discrimination of our quadrupole mass filter were made by comparing $50 \mathrm{eV}$ electron impact mass spectra of $n$-hexane taken on our instrument with those taken by ion cyclotron resonance, which has a known mass discrimination.

In the present studies the hydrogen line spectrum was the source of ionizing light. Monochromator slits were set at $100 \mu$, giving an optical resolution of about $1 \AA$ FWHM. All measurements were taken at a nominal average ion exit energy of less than $0.1 \mathrm{eV}$ to minimize translational energy effects on the ion molecule reactions studied.

The ion cyclotron resonance spectrometer used in this study has been previously described. ${ }^{10}$ Ion trapping studies were performed by the method of McMahon and Beauchamp, ${ }^{11}$ as previously described. All experiments on both instruments were conducted at ambient temperatures.

\section{RESULTS}

\section{A. Internal energy states populated near threshold}

The parent ion photoionization efficiency of vinyl fluoride is presented in Fig. 1(a). The ionization cross section shows an abrupt onset at $10.363 \pm 0.015$ $\mathrm{eV}$ photon energy and a series of steps in the photon energy range 10.4-11.2 eV. The vibrational states of the molecular ion that correspond to these steps may be assigned using the photoelectron spectrum of vinyl fluoride shown in Fig. 1(b). Figure 1(b), taken from a recent publication of Reinke et al. ${ }^{12}$ is in good agreement with an earlier spectrum published by Lake and Thompson. ${ }^{13}$ According to the analysis of both groups, the major vibration excited on ionization is the $\mathrm{C}=\mathrm{C}$ stretch, denoted as $\nu_{1}$. The other two vibrational modes shown in Fig. 1(b) are assigned to a C-F stretch
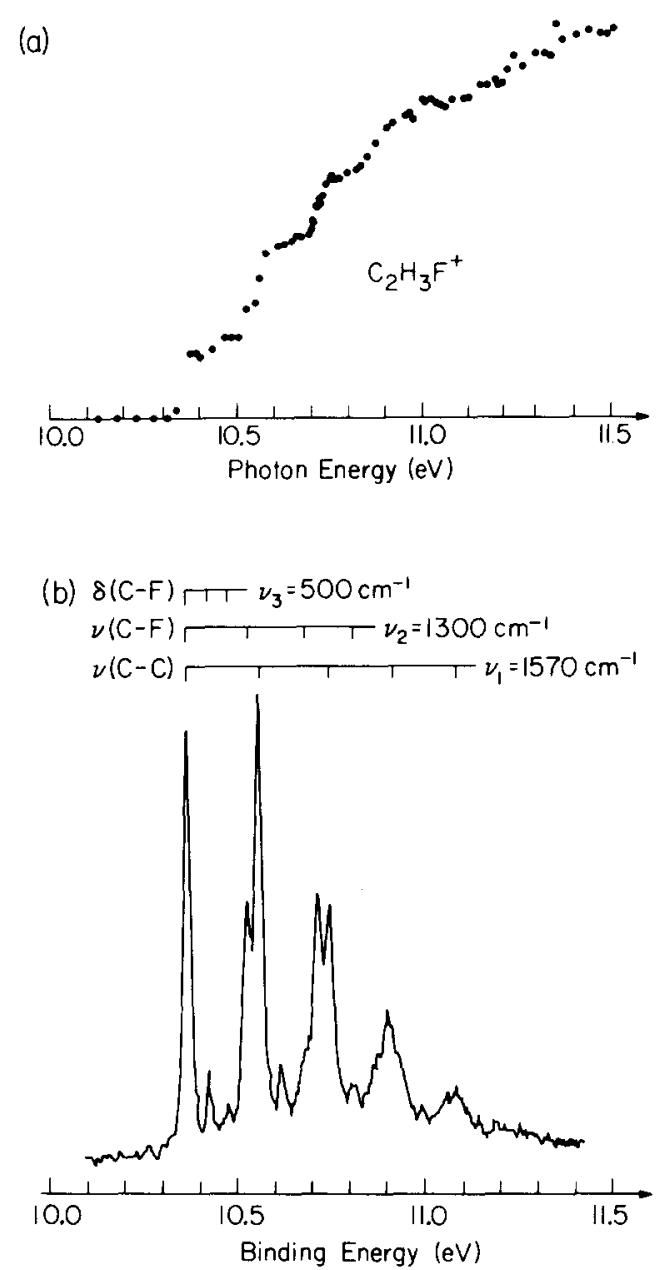

FIG. 1. Comparison of (a) the molecular ion photoionization efficiency of vinyl fluoride with (b) the photoelectron spectrum, taken from Ref. 12. There is good agreement between the two experiments concerning the thresholds and relative population of the lower vibrational levels of the molecular ion.

$\nu_{2}$ and an in-plane C-F bend $\nu_{3}$. Reinke et al. ${ }^{12}$ give frequencies of 1570,1300 , and $500 \mathrm{~cm}^{-1}$, respectively, for $\nu_{1}, \nu_{2}$, and $\nu_{3}$. The small steps due to combinations involving $\nu_{3}$ can be seen in the photoionization data. However, the spacing of lines in the exciting spectrum is not sufficiently dense to allow the states involving $\nu_{1}$ and $\nu_{2}$ to be distinguished. Thus, the steps chosen for use in this study and represented in Fig. 1 (a) do not always represent a single vibrational state but rather a combination of two or more states with a narrow internal energy spread. The specific population of states comprising each step may be estimated by comparing Figs. 1(a) and 1(b).

The photoionization mass spectrum of vinyl fluoride has also been studied by Reinke et al. ${ }^{14}$ using synchrotron radiation as an ionization source. Their data agree well with the data of Fig. 1(a) in the photon energy range common to both studies. However, at photon energies from 11.2 to $13 \mathrm{eV}$ they note a monotonic increase in the photoionization efficiency. Since the photoelectron spectrum shows no states accessible by direct ionization in this region, Reinke et al. ${ }^{12,14}$ attribute this structure to indirect ionization processes. 
Our raw data also show an increase in this photon energy region which can be attributed mostly to change in quantum efficiency of the sodium salycilate phosphor used in our study. When the data are corrected for the decrease in quantum efficiency of our scintillator, the rise with photon energy of our data becomes less dramatic. Our data indicate an increase in ionization efficiency of about $8 \%$ in the region from 11.5 to 12.0 $\mathrm{eV}$ which may be attributable to autoionization from a dense manifold of superexcited states in this region.

The lowest energy fragmentation process of the vinyl fluoride parent ion is loss of $\mathrm{HF}$ to form $\mathrm{C}_{2} \mathrm{H}_{2}^{*}$. We observe this fragmentation at photon energies above $13.31 \pm 0.05 \mathrm{eV}$. This is lower than the value of 13.51 $\pm 0.03 \mathrm{eV}$ found by Reinke et al. ${ }^{14}$ a discrepancy which we are unable to explain. Our value is also $1.16 \mathrm{eV}$ higher than the adiabatic appearance potential of 12.15 $\mathrm{eV}$ calculated from the heat of formation of $\mathrm{C}_{2} \mathrm{H}_{2}^{+} \mathrm{de}-$ rived from acetylene. The fact that the measured appearance potential of $\mathrm{C}_{2} \mathrm{H}_{2}^{+}$coincides with the second ionization potential of vinyl fluoride rather than the thermodynamic fragmentation threshold suggests that if autoionization occurs at photon energies above 12.15 $\mathrm{eV}$, the ions produced do not retain sufficient internal energy to dissociate.

Our study of Reactions (1)-(3) is restricted to photon energies from 10.35 to $11.25 \mathrm{eV}$. It is assumed that the parent ions in this region are formed primarily by direct ionization and obey the step function threshold law. This assumption is justified by the excellent correspondence between the photoionization efficiency and photoelectron spectrum in this energy range, as well as the lack of obvious structure due to autoionization. Ions produced by autoionization will have an unknown distribution of internal energies and cannot be treated by the methods used in this study. Since the thresholds for several fragments coincide with the second ionization potential of vinyl fluoride, ions formed in electronically excited states are expected to decompose into fragments on a time scale rapid with respect to ionmolecule collision times and thus be unavailable for reaction. This expectation is supported by the absence of a large increase in the parent ion photoionization efficiency above the threshold for the first electronically excited state of the ion. The expected increase in ionization cross section is restricted to fragmentions. ${ }^{14}$ In view of these factors, our primary interest in this work is the Franck-Condon manifold of the ground electronic state of the vinyl fluoride parent ion.

\section{B. Trapped ion ICR study of vinyl fluoride reactions}

Vinyl fluoride has not previously been studied using ICR trapped ion methods. Figure 2 presents trapped ion data on the vinyl fluoride system at $11.5 \mathrm{eV}$ electron energy, at which only the parent ion is formed. The qualitative features of the data are consistent with earlier studies. ${ }^{2-6}$ The parent ion undergoes Reactions (1)- (3) with an overall disappearance rate constant measured as $2.9 \times 10^{-10} \mathrm{~cm}^{3}$ molecule $\mathrm{e}^{-1} \cdot \mathrm{sec}^{-1}$. This number compares well with the value of 3.2 $\times 10^{-10}$ obtained by Sieck et al. ${ }^{6}$ using $10.6 \mathrm{eV}$ photons,

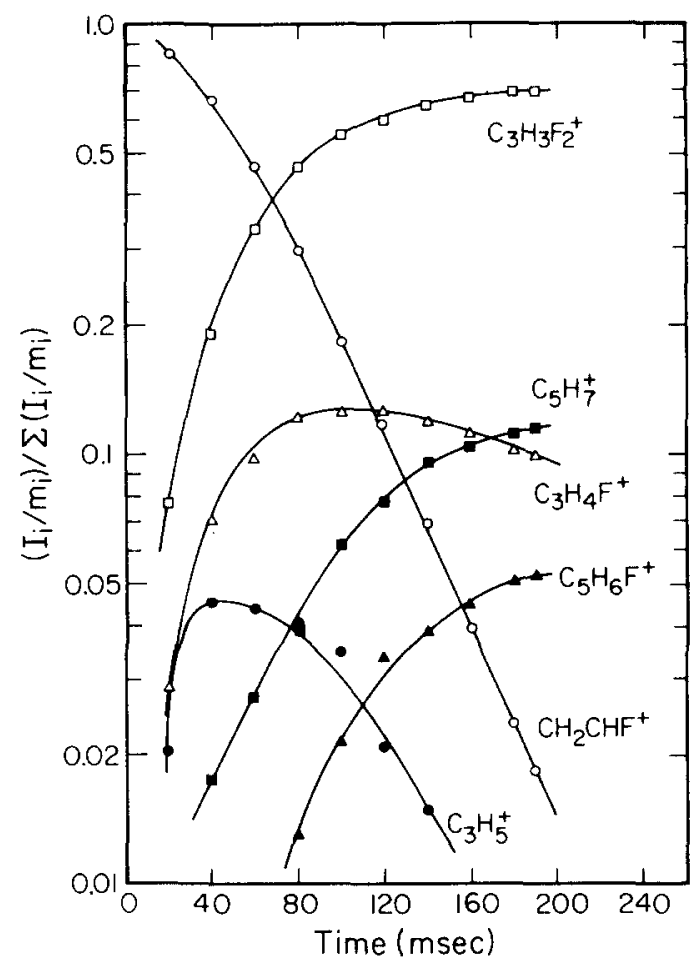

FTG. 2. Variation of ion abundance with time in $2.8 \times 10^{-6}$ torr of vinyl fluoride ionized at $11.5 \mathrm{eV}$. These data, obtained by ICR trapped ion techniques, yield a disappearance rate constant for $\mathrm{C}_{2} \mathrm{H}_{3} \mathrm{~F}^{*}$ of $2.9 \times 10^{-10} \mathrm{~cm}^{3}$ molecul $\mathrm{e}^{-1} \cdot \mathrm{sec}^{-1}$.

and is in fair agreement with the values of $2.1 \times 10^{-10}$ obtained by Anicich and Bowers ${ }^{5}$ and $2.2 \times 10^{-10}$ of McAskill and Harrison. ${ }^{3}$

As in previous studies, the products of Reactions (1) and (2) were found to react further by Processes (4) and (5). In

$$
\begin{aligned}
& \mathrm{C}_{3} \mathrm{H}_{5}^{+}+\mathrm{C}_{2} \mathrm{H}_{3} \mathrm{~F}-\mathrm{C}_{5} \mathrm{H}_{7} \mathrm{~F}^{+}+\mathrm{HF} \\
& \mathrm{C}_{3} \mathrm{H}_{4} \mathrm{~F}^{+}+\mathrm{C}_{2} \mathrm{H}_{3} \mathrm{~F} \rightarrow \mathrm{C}_{5} \mathrm{H}_{6} \mathrm{~F}_{2}^{+}+\mathrm{HF}
\end{aligned}
$$

calculating product distributions, the product yields in Reactions (4) and (5) are added to those of their precursors.

The data in Fig. 2 are indicative of the participation of excited states in the reaction kinetics. There is a reproducible curvature in the parent ion decay. In addition, the relative product yields for Reactions (1)-(3) change in the course of the reaction from $0.20: 0.24: 0.56$ at $15 \%$ conversion to $0.12: 0.17: 0.70$ at $99 \%$ conversion.

\section{Dependence of reaction cross sections on internal energy}

The photoionization efficiencies of the vinyl fluoride parent ion and two of the three secondary products are shown in Fig. 3. The third product ion, $\mathrm{C}_{3} \mathrm{H}_{4} \mathrm{~F}^{*}$, is omitted for clarity. These data, taken at less than $14 \%$ conversion to products, are normalized so that the three curves coincide at energies corresponding to the vibrational ground state of the parent ion. Given the assumption of step function threshold behavior, the heights of successive steps in the parent ion are proportional to the number of ions formed in the corre- 


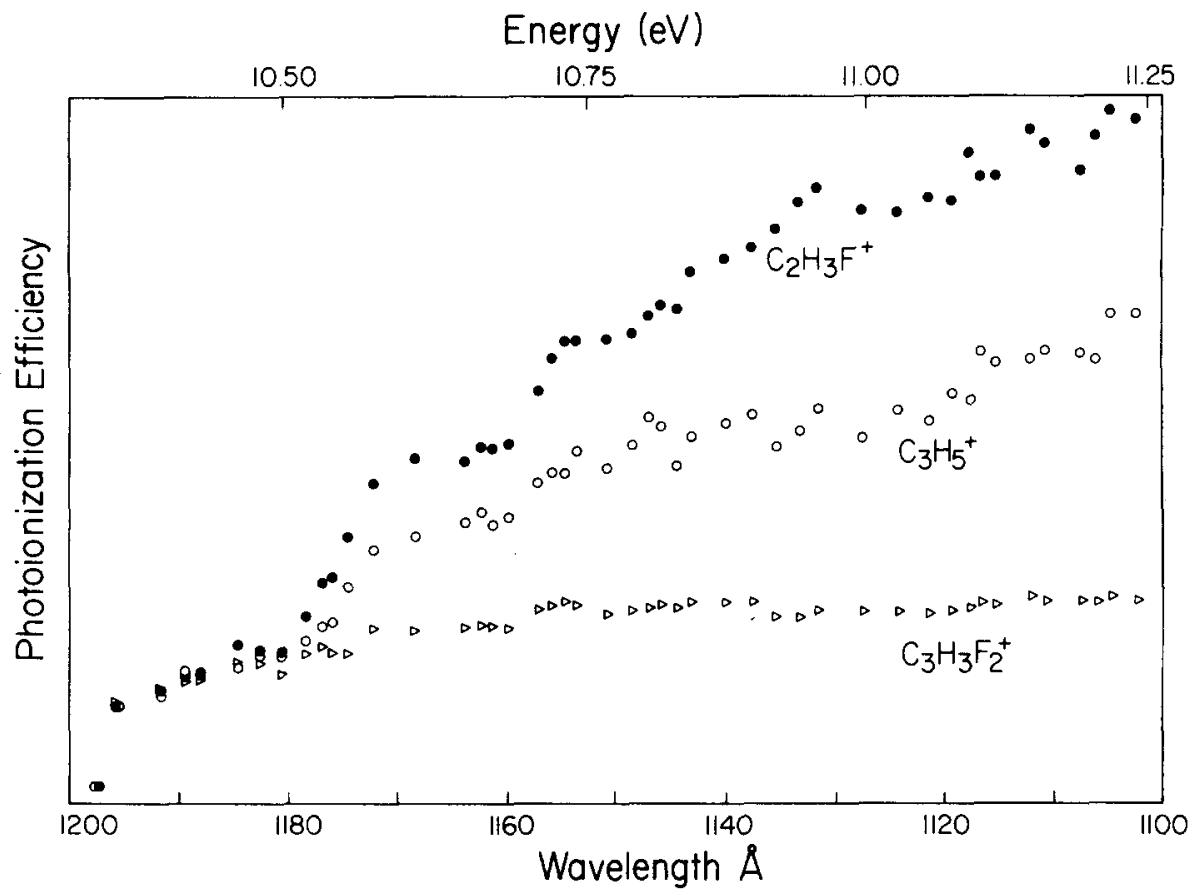

FIG. 3. Photoionization efficiency data for vinyl fluoride reactant ion and product ions $\mathrm{C}_{3} \mathrm{H}_{5}^{+}$and $\mathrm{C}_{3} \mathrm{H}_{3} \mathrm{~F}_{2}^{+}$. The third product ion, $\mathrm{C}_{3} \mathrm{H}_{4} \mathrm{~F}^{*}$, was omitted for clarity. Molecular ions in higher vibrational states exhibit decreased reactivity, particularly toward formation of the $\mathrm{C}_{3} \mathrm{H}_{3} \mathrm{~F}_{2}^{+}$product. sponding internal energy levels; the heights of successive steps in the product ions are proportional to the number of product ions formed by parent ions in the corresponding levels. For a particular state, the ratio of the product step height to the parent step height is proportional to the reaction cross section for parent ions in that state. Reaction cross sections obtained in this manner are summarized in Table $I$.

The data in Fig. 3 and Table I allow some immediate conclusions. First, the cross sections for all three reaction channels decrease with increasing internal energy in the reactant ion. Second, the minor products show the least change with increasing internal energy, while the major Reaction (3) is dramatically affected. The addition of as little as $0.18 \mathrm{eV}$ of vibrational energy to the parent ion reduces the probability of Reaction (3) by $75 \%$. There is no evidence that the particular modes of vibration have distinguishable effects on the reaction cross section. The important factor appears to be only the mean vibrational energy content, consistent with the hypothesis of extensive randomization of internal energy in the activated intermediate.

TABLE I. Relative cross sections for Reactions (1)-(3) for lower vibrational states of vinyl fluoride reactant ion.

\begin{tabular}{lllllll}
\hline $\begin{array}{l}\text { Step } \\
\text { number }\end{array}$ & $\begin{array}{l}\text { Major vibrational } \\
\text { states } \nu_{1} \nu_{2} \nu_{3}{ }^{b}\end{array}$ & $\begin{array}{l}\text { Mean internal } \\
\text { energy, eV }\end{array}$ & \multicolumn{4}{c}{ Relative cross sections } \\
& $\sigma_{1}$ & $\sigma_{2}$ & $\sigma_{3}$ & $\sigma_{\text {Total }}$ \\
\hline 1 & 000 & 0.00 & 0.086 & 0.121 & 0.792 & 1.000 \\
2 & 001,002 & 0.06 & 0.062 & 0.105 & 0.456 & 0.623 \\
3 & 010,100 & 0.18 & 0.060 & 0.100 & 0.159 & 0.319 \\
4 & $101,020,110,200$ & 0.35 & 0.048 & 0.066 & 0.135 & 0.249 \\
5 & $102,030,210,300$ & 0.53 & 0.037 & 0.039 & 0.052 & 0.128 \\
\hline \hline
\end{tabular}

${ }^{2}$ From Fig. 1(a).

bAssignments from Ref. 12: $\nu_{1}-\mathrm{C}=\mathrm{C}$ stretch; $\nu_{2}-\mathrm{C}-\mathrm{F}$ stretch; $\nu_{3}$ - in plane C-F bend. The list of states may not be exhaustive for each step.

Normalized relative to the total cross section for ground state reactants.
In order to make a qualitative comparison with earlier data, total product distributions at photon energies corresponding to vibrational plateaus are listed in Table II along with earlier results under differing experimental conditions. Photons of $10.4 \mathrm{eV}$ produce only ground state ions; higher energy photons form a distribution of

TABLE II. Product distribution at different ionization conditions.

\begin{tabular}{|c|c|c|c|c|}
\hline \multirow[b]{2}{*}{ Ionization source } & \multicolumn{3}{|c|}{ Product distribution } & \multirow[b]{2}{*}{$\begin{array}{l}\text { Apparent total } \\
\text { reaction rate }\end{array}$} \\
\hline & $\begin{array}{l}\text { Reaction } \\
\text { (1) }\end{array}$ & $\begin{array}{l}\text { Reaction } \\
\text { (3) }\end{array}$ & $\begin{array}{l}\text { Reaction } \\
\text { (3) }\end{array}$ & \\
\hline \multicolumn{5}{|l|}{ A. Present work } \\
\hline $10.40 \mathrm{eV}$ photons & 0.09 & 0.12 & 0.79 & 1.00 \\
\hline $10.50 \mathrm{eV}$ photons & 0.09 & 0.13 & 0.78 & 0.91 \\
\hline $10.65 \mathrm{eV}$ photons & 0.12 & 0.19 & 0.69 & 0.61 \\
\hline $10.75 \mathrm{eV}$ photons & 0.13 & 0.20 & 0.67 & 0.53 \\
\hline $10.85 \mathrm{eV}$ photons & 0.14 & 0.20 & 0.66 & 0.47 \\
\hline 11. $22 \mathrm{eV}$ photons & 0.16 & 0.21 & 0.63 & 0.41 \\
\hline \multicolumn{5}{|l|}{ B. Other studies } \\
\hline \multicolumn{5}{|l|}{ Sieck et al. ${ }^{\mathrm{b}}$} \\
\hline $10.6 \mathrm{eV}$ photons & 0.10 & 0.19 & 0.71 & $3.2 \times 10^{-10}$ \\
\hline $11.7 \mathrm{eV}$ photons & 0.26 & 0.27 & 0.46 & \\
\hline Anicich and Bowers $s^{c}$ & 0.09 & 0.18 & 0.72 & $2.1 \times 10^{-10}$ \\
\hline \multicolumn{5}{|l|}{ Herman and Harrison } \\
\hline "Second order" & 0.27 & 0.33 & 0.40 & $1.3 \times 10^{-11^{e}}$ \\
\hline "Third order" & 0.12 & 0.23 & 0.64 & $2.2 \times 10^{-10^{6}}$ \\
\hline \multicolumn{5}{|l|}{ McAskill and Harrison } \\
\hline "Second order" & 0.26 & 0.27 & 0.47 & $2.1 \times 10^{-11^{e}}$ \\
\hline "Third order" & 0.15 & 0.21 & 0.65 & $2.3 \times 10^{-10 e}$ \\
\hline \multicolumn{5}{|c|}{$\begin{array}{l}\text { }{ }^{2} \text { Represents reaction probability for low conversion. Present } \\
\text { results are normalized to unity for } 10.40 \mathrm{eV} \text { photons. Pre- } \\
\text { vious results are quoted rate constants in units of } \mathrm{cm}^{3} \text { mole- } \\
\text { cule }^{-1} \cdot \mathrm{sec}^{-1} \text {. A rough correspondence may be obtained by } \\
\text { multiplying present values by the total rate } 2.9 \times 10^{-10} \mathrm{~cm}^{3} \\
\text { molecule }{ }^{-1} \cdot \mathrm{sec}^{-1} \text { observed in the trapped ion ICR experiments. } \\
\text { Reference } 6 \text {. } \\
\text { 'Reference 5. Similar results given by Ref. } 4 \text {. }\end{array}$} \\
\hline
\end{tabular}


vibrational states. As is expected from the data in Table II, increasing the number of ions in vibrationally excited states causes the fraction of the $\mathrm{C}_{3} \mathrm{H}_{5}^{+}$and $\mathrm{C}_{3} \mathrm{H}_{4} \mathrm{~F}^{+}$products to increase at the expense of the $\mathrm{C}_{3} \mathrm{H}_{4} \mathrm{~F}_{2}^{+}$product. Some interesting comparisons may be made between product ratios from different published sources. For example, our product ratio at $10.65 \mathrm{eV}$ agrees well with that of Sieck $^{6}$ at $10.6 \mathrm{eV}$ and with the ICR results of Anicich and Bowers. ${ }^{5}$ The "second order" distribution in the work of Harrison's laboratory ${ }^{2,3}$ agrees with the $11.7 \mathrm{eV}$ value of Sieck et $a l .{ }^{6}$ but indicates a higher degree of excitation than is seen in our work. Since direct ionization to states with ionization energies above $11.2 \mathrm{eV}$ is not a favored process, the increase in the average excitation seen in these studies may be due to autoionization from superexcited neutral states in this energy region.

Previous studies indicate that collisional deactivation of excited vinyl fluoride ions is an efficient process. ${ }^{2,3,5}$ In order to investigate the effects of collisional relaxation for ions in well characterized states, a study of the effect of pressure on Reactions (1)-(3) was performed. Variation of the fractional abundance of the $\mathrm{C}_{3} \mathrm{H}_{3} \mathrm{~F}_{2}^{+}$ product of Reaction (3) with vinyl fluoride pressure is shown in Fig. 4 for two photon energies. Ions formed at $10.41 \mathrm{eV}$ photon energy are in the vibrational ground state. The $10.64 \mathrm{eV}$ data correspond to the plateau of Step 3 [Fig. 1(a) and Table I]. By comparing the photonization and photoelectron data in Fig. 1, the population of specific vibrational states formed at 10.64 $\mathrm{eV}$ is found to be: $32 \%$ ground state, $12 \% \nu_{3}=1$ or 2 , $20 \% \nu_{2}=1$, and $36 \% \nu_{1}=1$. Thus, $68 \%$ of the ions are formed with a quantum of vibrational excitation, with a mean vibrational energy of $1350 \mathrm{~cm}^{-1}$ or $0.17 \mathrm{eV}$. Since the cross section for Reaction (1) decreases by $75 \%$ for this distribution of excited ions, they may be distinguished from ground state ions by their reaction kinetics. The data were fit to the reaction scheme

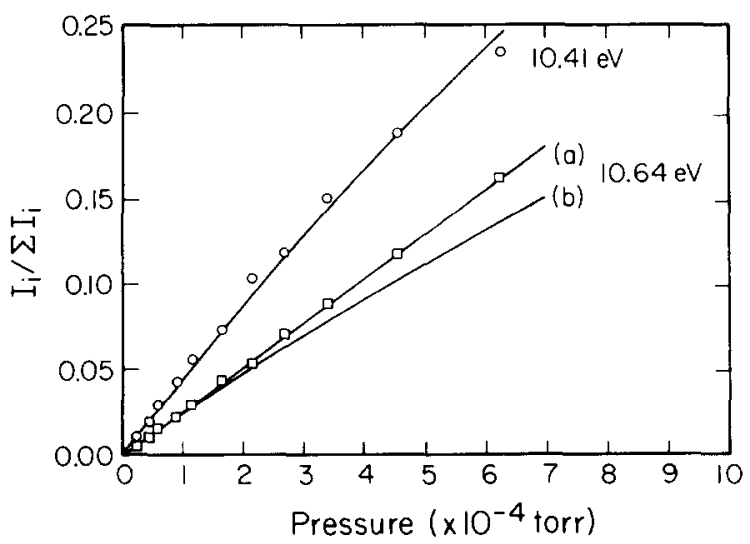

FIG. 4. Fractional aboundance of $\mathrm{C}_{3} \mathrm{H}_{3} \mathrm{~F}_{2}^{*}$ product of Reaction (3) as a function of vinyl fluoride pressure for two photon energies corresponding to Steps (1) and (3) in the ionization efficiency of the parent ion shown in Fig. 1(a). The lines represent fits to the data using the relative reaction efficiencies shown in Table I, as discussed in the text. Curves calculated for the 10.64 eV data are shown for (a) $k_{d}=4.1 \times 10^{-10} \mathrm{~cm}^{3}$ molecule $\mathrm{e}^{-1}$ $\cdot \sec ^{-1}$ and (b) $k_{d}=0$. shown below. Using relative product distributions from Table I, $k_{1}, k_{2}$, and $k_{4}$ were calculated relative to $k_{3}$, which was fit using the $10.41 \mathrm{eV}$ data, in which only ground state

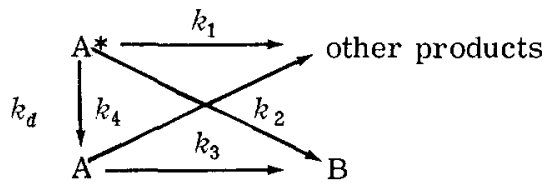

$$
\begin{aligned}
& \mathrm{A}=\mathrm{C}_{2} \mathrm{H}_{3} \mathrm{~F}^{+} \\
& \mathrm{B}=\mathrm{C}_{3} \mathrm{H}_{3} \mathrm{~F}_{2}^{+}
\end{aligned}
$$

reactants are present. The $10.64 \mathrm{eV}$ data were then fit by using the experimental distribution of initial states and adjusting $k_{d}$ by a least squares method. As a result $k_{d}$ was found to be $1.76 \pm 0.15$ times the value of $k_{3}$. Using $k_{3}=2.3 \times 10^{-10} \mathrm{~cm}^{3}$ molecule ${ }^{-1} \cdot \mathrm{sec}^{-1}$ from our ICR trapped ion data, we obtain $k_{d}=4.1 \pm 0.4 \times 10^{-10}$ $\mathrm{cm}^{3}$ molecule $\mathrm{e}^{-1} \cdot \mathrm{sec}^{-1}$. Fits to the $10.64 \mathrm{eV}$ data using the optimized $k_{d}$ and $k_{d}=0$ are shown in Fig. 4 .

The only comparable calculations have been those of Anicich and Bowers ${ }^{5}$ who used a model analogous to the one presented above (Mechanism Ia of Ref. 5) with the exception of their assumption that all ions are originally formed in the state $\mathrm{A}^{*}$. This model yields a deactivation rate of $3.5 \times 10^{-10} \mathrm{~cm}^{3}$ molecule ${ }^{-1} \cdot \mathrm{sec}^{-1}$ for the data of McAskill and Harrison. ${ }^{3}$ In view of the much higher degree of initial excitation in their system, it is somewhat surprising that their rate is in such good agreement with the value measured in the present work.

It is instructive to note that the $10.64 \mathrm{eV}$ data could also be fit by a simple pseudo-first-order model with an effective rate of 0.52 times $k_{3}$, or $1.19 \times 10^{-10} \mathrm{~cm}^{3}$ molecule $\mathrm{e}^{-1} \cdot \mathrm{sec}^{-1}$. This provides an excellent example that an approximately linear relationship between the fractional abundance of a product and pressure does not in itself guarantee a second order process.

\section{DISCUSSION}

The extreme sensitivity of Reactions (1)-(3) to internal excitation of the reactant ion explains much of the variation in the reported kinetics and product distribution of this system. ${ }^{2-5}$ Past studies invariably populated a distribution of vibrational states of the parent ion. Thus, reaction rates measured at low conversion represent an average of the individual cross sections given in Table I weighted by the relative populations of the internal states. At higher conversions the population of states changes continually due to reaction and collisional deactivation. As a result the kinetics depart from pseudo-first order, and the product distribution changes with the extent of reaction. These effects are clearly seen in the data of Herman and Harrison, ${ }^{2}$ McAskill and Harris on, ${ }^{3}$ and Sieck $e t$ al. ${ }^{6}$ The similarity in rate constants for relaxed ions determined by those workers with those of the present study is gratifying, as is the agreement in the deactivation rates calculated for our study and those calculated ${ }^{5}$ for the data of McAskill and Harrison. ${ }^{3}$

It is not completely clear why the earlier ICR studies ${ }^{4,5}$ could not be fit by the model of Anicich and Bowers ${ }^{5}$ without the assumption that essentially all ions were in their ground state. Certainly, the ICR product dis- 
tributions indicate a low degree of excitation. However, we feel there are indications that excited states do participate in the ICR experiment. First, our ICR trapped ion data, with a longer time scale and higher conversion does show a changing reactivity with time. Secondly, $O^{\prime}$ Malley and Jennings ${ }^{4}$ do report a change in product ratios with electron energy, indicating a changing vibrational population. Finally, we note that pure second order kinetics are not guaranteed by apparent linearity in the growth of product ions at low conversion, as mentioned previously in connection with Fig. 4.

Some differences may be noted between the vinyl fluoride system and the analogous reaction in ethylene. ${ }^{7}$ Primarily, the available states of the molecular ion differ for the two systems. At $13 \mathrm{eV}$ photon energy $70 \%$ of the ethylene molecular ions formed are initially in the ${ }^{2} B_{3}$ first excited state. In vinyl fluoride the thermodynamic appearance potential for the lowest energy fragmentation lies below the first electronically excited state, and the parent ion efficiency curve does not exhibit the dramatic increase in this state seen in the ethylene system. This indicates that most if not all electronically excited vinyl fluoride ions decompose before reaction can occur. As a result, when moderate $(>15 \mathrm{eV})$ ionizing energies are used the effects of vibrational energy remain those associated with the ground electronic state in vinyl fluoride, while the major effects noted in ethylene are due to ions formed in the first electronically excited state (where internal conversion to highly vibrationally excited ground state ions may precede reaction).

A second quantitative difference in the two systems is the greater sensitivity to internal excitation of the vinyl fluoride system, particularly Reaction (3). One factor that may be of importance here is the different reaction efficiencies for ground state ions in the two systems. Ion molecule reactions in ethylene occur on almost every collision, whereas in vinyl fluoride the reaction rate is only $19 \%$ of the collision rate. ${ }^{15}$ One possible explanation assumes that in each system the initially formed intermediate must rearrange before any product channels are available. If the ratio of decomposition rate to rearrangement rate is higher in the vinyl fluoride case than in ethylene, the relative reaction efficiencies follow. Added internal energy would further increase the decomposition rate in both systems, but if the rearrangement is initially much faster than decomposition in ethylene (as appears to be the case) then the vinyl fluoride system would be more sensitive to this decrease in lifetime of the initially formed complex.

Some insight into the mechanism of Reactions (1)-(3) is provided by recent studies. In a comprehensive study of reactions in mixtures of different fluoroethylenes, Anicich and Bowers ${ }^{15}$ present convincing evidence that the reactions in these systems involve cyclobutane intermediates that may open to a tetramethylene structure, rearrange by $\mathrm{H}$ or $\mathrm{F}$ atom migration, and eliminate a $\mathrm{C}(\mathrm{H}, \mathrm{F})_{3}$ radical to form products. One such system in their study is Reactions (6)-(9) in a mixture of ethylene and cis- or trans-1, 2 difluoroethylene. This sys- tem produces both the reactants and

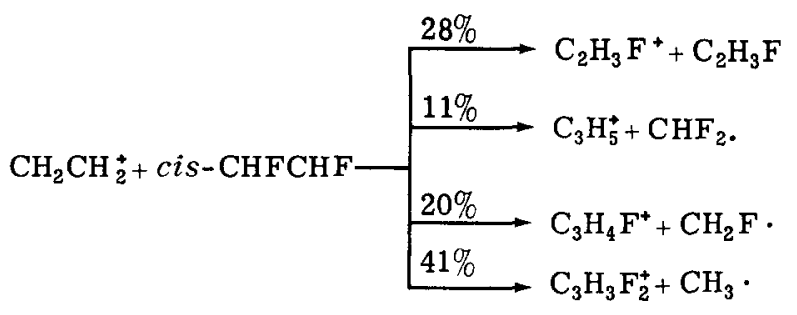

products of Reactions (1)-(3) in vinyl fluoride, suggesting that the cyclobutane intermediate I may be common to both systems. In the vinyl fluoride system intermediate II is also possible. Anicich and<smiles>F[C@H]1CC[C@@H]1F</smiles>

I<smiles>[CH+]1C[CH-]C1</smiles>

II
Bowers ${ }^{15}$ report studies of Reactions $(6)-(9)$ with ethylene- $d_{4}$ as a reactant. The $C_{3}$ products contained all possible isotopic combinations, including two $\left(\mathrm{C}_{3} \mathrm{H}_{2} \mathrm{D}_{3}^{+}\right.$and $\left.\mathrm{C}_{3} \mathrm{D}_{3} \mathrm{~F}_{2}^{+}\right)$which could not arise from a single atom migration. However, no isotopic scrambling occurs in the vinyl fluoride product of Reaction (6). These findings indicate that the decomposition of intermediate I, while complex, is still more specific than a total scrambling model would indicate.

While our data do not implicate specific intermediates or mechanisms for Reactions (1)-(3), several related studies may be proposed which would yield a more definitive understanding of this system. In our earlier study of ethylene ${ }^{7}$ it proved illuminating to compare the effects of internal energy on the decomposition modes of the $\left[\mathrm{C}_{4} \mathrm{H}_{8}^{+}\right]^{*}$ intermediate with the distribution of products observed for the decomposition of cyclobutane molecular ions formed with reasonably well characterized internal energy. Likewise it would be helpful to conduct a charge exchange or photoionization mass spectrometric study of the fragment distribution of the 1,2- and 1,3-difluorocyclobutanes as a function of internal energy. These data could be related to the product distributions of intermediates I and II, respective$l y$, as functions of internal energy. An independent study of intermediate I formed by an ion-molecule reaction pathway involves study of Reactions (6)-(9) by the methods used in the present work, varying internal energy in either ethylene or difluoroethylene as the ionic reactant. Use of isotopically labeled reactants may yield information on the barrier to specific $\mathrm{H}$ or $F$ atom migrations.

\footnotetext{
* This research was supported in part by the United States Energy Research and Development Administration under Grant No. AT (04-3) 767-8 and presents one phase of research carried out at the Jet Propulsion Laboratory, California Institute of Technology, under Contract No. NAS 7-100 from the National Aeronautics and Space Administration. The PIMS instrumentation was made possible by a grant from the President's Fund of the California Institute of Technology. tCamille and Henry Dreyfus Teacher-Scholar, 1971-1976.
} 
${ }^{1}$ From Ref, 5 and sources listed therein. The value of $\Delta H_{f}^{298}$ $\left(\mathrm{C}_{3} \mathrm{H}_{5}^{+}\right)$is assumed to be $224 \pm 2 \mathrm{kcal} / \mathrm{mole}$, from $\mathrm{S}$. E. Buttrill, A. D. Williamson, and P. LeBreton, J. Chem. Phys. 62,1586 (1975).

${ }^{2}$ J. A. Herman and A. C. Harrison, Can. J. Chem. 47, 957 (1969).

${ }^{3}$ N. A. McAskill and A. C. Harrison, Int. J. Mass Spectrom. Ion Phys, 5, $193(1970)$.

${ }^{4}$ R. M. O'Malley and K. R. Jennings, Int. J. Mass Spectrom. Ion Phys. 2, 441 (1969).

${ }^{5}$ V. G. Anicich and M. T. Bowers, Int. J. Mass Spectrom. Ion Phys. 12, 231 (1973).

${ }^{6}$ L. W. Sieck, R. Gorden, S. G. Lias, and P. Ausloos, Int. J. Mass Spectrom. Ion Phys. 15, 181 (1974).

${ }^{7}$ P. R. LeBreton, A. D. Williamson, J. L. Beauchamp, and W. T, Huntress, J. Chem. Phys. 62, 1623 (1975).

${ }^{8}$ W. A. Chupka, "Ion-Molecule Reactions by Photoionization
Techniques," in Ion Molecule Reactions, edited by J. L. Franklin (Plenum, New York, 1972).

${ }^{9}$ K. Watenabe, F. M. Matsunaga, and H. Sakai, Appl. Opt. 6, 391 (1967).

${ }^{10}$ M. S. Foster and J. L. Beauchamp, J. Am. Chem. Soc. 97. 4808 (1975).

${ }^{11}$ T. B. Mc Mahon and J. L. Beauchamp, Rev. Sci. Instrum. 43. 509 (1972).

${ }^{12}$ D. Reinke, H. Baumgärtel, T. Cvitas, L. Klasinc, and $H$. Güsten, Ber. Bunsenges. Phys. Chem. 78, 1145 (1974).

${ }^{13}$ R. F. Lake and H. Thompson, Proc. R. Soc. London A 315, 323 (1970).

${ }^{1}$ D. Reinke. R. Kraessig, and H. Baumgärtel, Z. Naturforsch. A 28, 1021 (1973).

${ }^{15}$ V. G. Anicich and M. T. Bowers, Int. J. Mass Spectrom. Ion Phys, 13, 359 (1974). 